\title{
Trust Evaluation by Mining of E-commerce Feedback Comments
}

\author{
Priyanka Kumbhar ${ }^{1}$, Manjushri Mahajan ${ }^{2}$ \\ ${ }^{1,2}$ Savitribai Phule University of Pune, Pune, G. H. Raisoni College of Engineering, Wagholi, Pune, Chandannagar, Pune, India
}

\begin{abstract}
In E-commerce applications trust and reputation are decisive factors in order to rate the sellers or products. Reputation Systems used in e-commerce have the main aim is to guide the users in a proper way so that they can choose between the different available sellers on E-commerce. In reputation system the models are meant to project the different sellers as per their services and quality they provide to users. Different Models differ between each other by the methods they opt to calculate the trustworthiness measure for different sellers. Review mining or Feedback mining method of trust calculation will be a very good idea as the users are free to express themselves in textual feedback comments. So here in our proposed work we have given a multidimensional trust model for computing reputation scores from user reviews. In order to perform this functionality we presented an algorithm for mining feedback comments for dimension ratings and weights. In this algorithm we are using techniques like NLP, Topic Modeling.
\end{abstract}

Keywords: E-commerce, Natural Language Processing, Ontology Language, Topic Modeling etc.

\section{Introduction}

There has been a tremendous growth in e-commerce applications such as Amazon, Flipkart. On this site buyers and sellers conduct transactions over the internet. Customers are attracted to E-commerce sites not only due to the convenience in accessing the information of items on-sold but also because of the availability of other buyers feedback on the purchased items related to different aspects[1]. There are many E-commerce websites encourage buyers to provide feedback, some web sites allow users to provide feedback in the form of rating with some textual comments so that the other customer can review this comments.

Reputation calculation systems have been implemented in online shopping sites systems such as eBay, Flipcart and Amazon (for third-party sellers), In this systems the overall reputation trust scores for sellers are computed by aggregating feedback ratings. Most of the reputation systems are related to the ratings that a seller received from customers. Users provide this ratings on the basis of services they got from the seller. Means ratings indicate the ability of the seller to provide satisfactory transactions in the future. These rating are beneficial to new buyers. In E-commerce site like Ebay the reputation score for a seller is computed by aggregating buyer feedback ratings in the past 12 months [2]. They calculate this rating by total number of positive ratings minus the total number of negative ratings or the percentage of positive ratings out of the total number of positive ratings and negative ratings given by buyers.

The output of this kind of reputation system is mostly average and from the buyer's perspective the average rating may not be a fully reliable measure [2]. Another concern here is that browsing through tens of pages of comments can be time consuming and a hectic task to be carried out. So here we need a better reputation system to represent the reputation of seller accurately. We can also refer this reputation as trust. We can define the trust as "the extent to which one party measures the other party's willingness and ability to act in the measuring party's interest".
The motivation of our proposed work is that online feedback comments contain distinct information for users to rank sellers according to the services they have provided to us [11]. So we can use the content of comments to reliably evaluate the trustworthiness of e-commerce sellers. By mining the wealth of information in feedback comments we can extract embedded opinions towards different aspects of transactions related to particular seller and based on this calculate comprehensive reputation profiles for sellers. In this work using the positive and negative subjectivity of opinion towards aspects of transactions as dimension ratings to calculate the Comment-based Multi-dimensional trust (that is a fine-grained multi-dimension trust evaluation model for online shopping sites.

Our main objective is to provide a comprehensive trust profiles for sellers that allows buyers to conduct their online shopping based on past experience. We are mainly working on extracting dimension ratings from feedback comments and further aggregating these dimension ratings to compute dimension trust scores of sellers.

\section{Literature Review}

There are various researches that have been done related to reputation calculation. Some of the works are presented below. Authors X. Wang, L. Liu In [12] has presented open environments trust relationship which is build using ratings. Customer Ratings are also be called as recommendations or feedbacks provided by uses. There are many Rating aggregation algorithms are used to build up trust relationship for sellers by using rating aggregation algorithms. As other Complex methods and algorithms are not always cost effective and resistant to fake ratings provided by buyers. One of the system named Review aggregator is one such system using rating aggregation algorithm given by $P$. Thomas and D. Hawking, in [3]. First it stores different reviews and makes use of these to support websites where the users can read this reviews. They have assign each review a numeric value based on the positive polarity expressed in that particular review and based on that an average assessment is made. 


\section{International Journal of Science and Research (IJSR) \\ ISSN (Online): 2319-7064 \\ Index Copernicus Value (2013): 6.14 | Impact Factor (2014): 5.611}

Authors H. Zhang, Y. Wang presented [5] a Peer Trust framework used in peer to peer systems. Here they have used contextual factors for computing trust scores and weights for different peers. The contextual factors includes transaction item details, item transaction amount and transaction time. The first term Transaction item refers to the product in traded in a transaction second the properties of the item like product qualities, product categories of which determine the nature of the transaction. Third term Transaction amount refers to sum of prices of all products in a transaction done by a particular user at a specific time. Higher the transaction amount more is the chance for fraud to happen. Here the term Transaction time refers to time when a transaction happens. While calculating trust Transaction time has a specific feature. Here the consideration is that any query on temporal dimension should start from a previous point (e.g. one week ago) and end at present time.

The main disadvantage of this work is that it uses a bit large amount of data space as well as computation time. Another limitation is flexibility while considering the contextual factors because the factors are chosen while the system is designed. So the output of this system is that, the exact ranking of the sellers cannot be ensured.

EigenTrust algorithm proposed by S. D. Kamvar and M. T. Schlosser [6] uses rating matrix representation for local trust scores and calculates the global ratings for peers from the rating matrix. This system is a reputation management algorithm for peer to peer Systems. In this work each peer in the network assigned a unique global trust value based on peer history of uploads and reduce the number of unauthenticated files in peer to peer network. They have consideration here that if peer a trust peer $b$, then all peers trusted by $b$ can also be trusted a. Here they compute the local trust value for all peers that have provided it with authenticate or fake downloads based on the satisfactory or unsatisfactory transactions that it had.

The main limitation of this system is that it assumes that feedback ratings are given already and the aggregation algorithms are given priority.

Another concept come into the picture such as Opinion mining is also called sentiment analysis which is a part of natural language processing and computational linguistics

which identify and extract subjective information [7] from the source like comments, reviews. The main part of these systems is text analysis. Here the concept opinion mining targets to determine the polarity of a document with respect to some context of the text. Further it determines the different opinions expressed by different authors about some topics. The methods used for Accuracy measurement are precision or recall functions.

Aspect opinion extraction is a concepts presented in various papers. Several existing work on aspect opinion mining on product reviews and movie reviews [8]-[10]. Out of these M. $\mathrm{Hu}$ and $\mathrm{B}$. Liu in [8] used frequent nouns and noun phrases as aspects for product reviews and further opinion lexicon is developed to identify opinion orientations. Authors G. Qiu and B. Liu [9] have further improved the previous methods to apply lexical knowledge patterns to improve the aspect extraction accuracy. And next in [10] dependency relation parsing is used to mine aspect opinions for movie reviews. Here they have not group the aspect opinion expressions into clusters.

Unsupervised topic modeling approaches are presented by some authors which is used to jointly model opinions and aspects . They have considered here the probabilistic Latent Semantic Analysis model [9]. There are various models presented related to this which differ in granularities and how aspects and opinions interactions with each other [14], [15].

There are another approach for text mining is ontology language. It is beneficial to natural language processing which here would be used for feedback comments mining[16]. Now a days ontology is an important component for information system and information processing. So ontology languages can be used for processing of text in recommendation system.

In all these trust or reputation models however, weightings for dimension trust are either not considered or assumed given.

\section{Implementation Details}

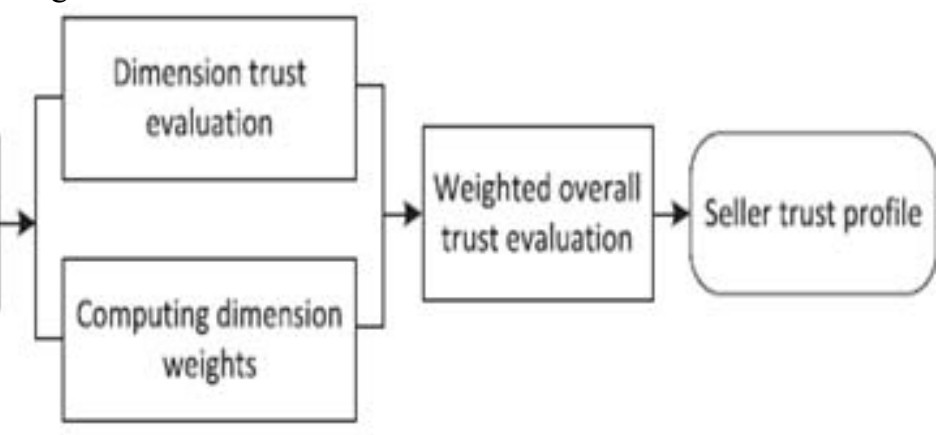

Figure: System Architecture

Our approach consisting of various concepts, First we describe our approach based on the typed dependency analysis to extracting aspect opinion expressions and identifying their associated ratings. Then on these dimension expressions we implement our LDA based algorithm for clustering dimension expressions into dimensions and computing dimension weights [1]. 


\section{International Journal of Science and Research (IJSR) \\ ISSN (Online): 2319-7064}

Index Copernicus Value (2013): 6.14 | Impact Factor (2014): 5.611

Type dependency relation parsing [12][13] is a method where we can represent a sentence as a set of dependency relations between pairs of words in the form of (head, dependent) expressions, In this content words are chosen as heads whereas other related words depend on the heads. Consider an example In order to parse this we are using standard library named Standford NLP parser.

The output of NLP parser is dependency relations expressing grammatical relationships. Out of these grammatical relations, we select the relations that express the modifying relation first adjectives and nouns, second adverbs and verbs. The adjectives, verbs, nouns are already we get through standford parser. As we gone through the comments we can say that the modifying relations generally the noun or verb expresses the target concept under consideration, and the adjective or adverb expresses opinion towards the target concept. So here we are representing the modifying relations as (modifier, head) pairs. These pairs can be called as Dimension expression in our work.

Further from these dimension expressions we are looking to find out the ratings towards the head terms are identified by identifying the prior polarity of the modifier terms by SentiWordNet library, which is a public opinion lexicon library. The various polarities given by Sentiwordnet are positive, negative and neutral. These polarities can be represented as ratings of $+1,-1$ and 0 .

Next we used LexicalLDA algorithm in order to cluster aspect expression in Dimensions. Here we called this algorithm as Lexial LDA because we are providing input to the LDA as dimension expressions which are in the form of (Modifier, Head).

\section{Conclusion}

We have proposed a multi-dimensional trust evaluation model for computing comprehensive trust profiles for sellers in online shopping sites. Here we compute dimension trust scores and dimension weights automatically via extracting dimension ratings from feedback comments and aggregating with feedback rating. Based on this trust scores we can distinctively identify the reputable sellers from another seller that have had bad history with previous buyers. Another important thing here that we have overcome is "All good reputation" as the ratings are more reasonable and acceptable not like all sellers have high scores. So our proposed work can significantly reduce the strong positive bias in eCommerce reputation systems. This model is good assistance to the buyers when doing online shopping in order to get prevented from being a victim of fraud and untrusted sellers.

\section{Acknowledgement}

I would like to express my thanks to my guide Prof. Manjushri Mahajan for his highly appreciable support and encouragement also to my HOD Prof. P.V.Gupta. Their guidance is a force behind the completion of this paper. I am grateful for all the suggestions and hints provided by him. My acknowledgment of gratitude to all who supported to make it possible.

\section{References}

[1] Xiuzhen Zhang, Lishan Cui, and Yan Wang, "Computing Multi-Dimensional Trust by Mining E-Commerce Feedback Comments " IEEE TRANSACTIONS ON KNOWLEDGE AND DATA ENGINEERING VOL:26 NO:7 YEAR 2014

[2] P. Resnick and E. Friedman, "Reputation Systems: Facilitating Trust in Internet Interactions," Communications of the ACM, vol. 43, pp. 45-48, 2000

[3] P. Thomas and D. Hawking, "Evaluation by comparing result Set in context," in Proc. 15th ACM CIKM, Arlington, VA, USA, 2006, pp. 94101. Department

[4] X. Wang, L. Liu, and J. Su, "RLM: A general model for Trust representation and aggregation," IEEE Trans. Serv. Comput., vol. 5, no. 1, pp. 131143, Jan-Mar, 2012.

[5] H. Zhang, Y. Wang, and X. Zhang, "Efficient contextual transaction trust computation in e-commerce environments," in Proc. 11th IEEE TrustCom, Liverpool, UK, 2012.

[6] S. D. Kamvar, M. T. Schlosser, and H. Garcia-Molina, "The EigenTrust algorithm for reputation management in P2P networks," in Proc. 12th Int. Conf. WWW, Budapest, Hungary, 2003.

[7] G. Qiu, B. Liu, J. Bu, and C. Chen, "Opinion word expansion and arget extraction through double propagation," Comput. Linguist, vol. 37, no. 1, pp. 927, 2011.

[8] M. Hu and B. Liu, "Mining and summarizing customer reviews," in Proc. the fourth Int. Conf. on KDD, 2004, pp. $168-177$.

[9] G. Qiu, B. Liu, J. Bu, and C. Chen, "Opinion word expansion and target extraction through double propagation," Computational linguistics, vol. 37, no. 1, pp. 9-27, 2011.

[10] L. Zhuang, F. Jing, X. Zhu, and L. Zhang, "Movie review mining and summarization," in Proc. the 15th ACM Int. Conf. on Information and knowledge management, 2006, pp. $43-50$.

[11] J. O'Donovan, B. Smyth, V. Evrim, and D. McLeod, "Extracting and visualizing trust relationships from online auction feedback comments," in Proc. IJCAI'07, 2007, pp. 2826-2831.

[12] M. De Marneffe, B. MacCartney, and C. Manning, "Generating typed dependency parses from phrase structure parses," in Proc. LREC, vol. 6, 2006, pp. 449-454.

[13] M. De Marneffe and C. Manning, "The stanford typed dependencies representation," in Proc. the workshop on Cross- Framework and Cross-Domain Parser Evaluation, 2008.

[14] S. Brody and N. Elhadad, "An unsupervised aspectsentiment model for online reviews," in Proc. Human Language Technologies: Chapter of the Association for Computational Linguistics, 2010, pp. 804.

[15] W. X. Zhao X. Li, "Jointly modeling aspects and opinions with a MaxEnt-LDA hybrid," in Proc. the 2010 Conference on Empirical Methods in Natural Language Processing, 2010, pp. 56-65.

[16] Shabina Dhuria, Dr. Harmunish Taneja, "Ontology Equipped Natural Language Processing for Real World Applications", International Journal of Advanced Research in Computer Science and Software Engineering Volume 4, Issue 4, April 2014 